\title{
2.4 GHz wake-up receiver with suppressed substrate noise coupling
}

\author{
Shota Hiramatsu ${ }^{1} \cdot$ Masayuki Ikebe $^{1}$ (D) $\cdot$ Eiichi Sano ${ }^{1}$ \\ Received: 5 October 2017 / Revised: 7 December 2018/Accepted: 2 April 2019/Published online: 15 April 2019 \\ (C) The Author(s) 2019
}

\begin{abstract}
The switching noise generated in digital circuits propagates through conductive silicon substrate to analog circuits in a mixed-signal CMOS LSI. Substrate noise coupling may degrade the performance of the analog circuits, and may result in a fault operation of the mixed-signal LSI in the worst-case. In this paper, the substrate noise coupling between the clock recovery circuit and the input port of the envelop detector in a low-power wake-up receiver (WuRx) was investigated experimentally. The propagation path of the substrate noise coupling was clarified by comparing the experimental results with the circuit simulations on the basis of an equivalent circuit model. The design of the WuRx was modified on the basis of the findings to suppress the substrate noise coupling. The fabricated WuRx successfully operated a 100-kbps PWM signal with a carrier frequency of $2.4 \mathrm{GHz}$, and the effectiveness of the noise coupling suppression recipe was confirmed.
\end{abstract}

Keywords Wake-up receiver $\cdot$ Mixed signal $\cdot$ Analog and digital $\cdot$ Substrate noise coupling $\cdot$ Clock and data recovery

\section{Introduction}

Reducing power consumption in sensor nodes is a key issue in the development of the Internet-of-Things. A candidate to achieve low power consumption is a wake-up radio. A wake-up receiver (WuRx) continuously monitors the wireless-communication channel for receiving communication requests from other sensor nodes. When the WuRx receives a communication request, it activates other parts of the sensor node for sensing, data processing, and data transmission. When not activated, these parts remain in sleep mode to save the power consumption. Many kinds of WuRx with wireless carrier frequencies from 0.9 to $60 \mathrm{GHz}$ have been reported [1-7].

In the microwave and millimeter-wave regions, input impedance matching is essential. When the input characteristic impedance of an analog frontend circuit does not match the characteristic impedance of the transmission line connected to the antenna in wireless equipment, the RF power input to the analog frontend circuit is reflected and the effective input power is reduced. Several kinds of

Masayuki Ikebe

ikebe@ist.hokudai.ac.jp

1 Research Center for Integrated Quantum Electronics, Hokkaido University, Sapporo 060-0813, Japan impedance matching technique are used in the microwave and millimeter-wave regions. In hybrid integration systems, bulk acoustic wave (BAW) and surface acoustic wave (SAW) filters are good candidates for RF input [2]. On the other hand, an impedance matching circuit monolithically integrated with CMOS IC is desired to reduce the cost and size of sensor nodes. In millimeter-wave regions, like $60 \mathrm{GHz}$, a microstrip line with a bottom-layer metal for the ground plane and a top-layer metal for the signal line is commonly used in the impedance matching circuit [5]. In the microwave region, a microstrip line becomes unrealistically long and on-chip spiral inductors are used to reduce the chip sizes of CMOS ICs [3, 4, 6]. A usual spiral inductor is composed of a top-layer metal without a lowerlayer metal for the ground plane because the Eddy current flowing in the lower-layer metal degrades the quality factor of the inductor.

When the logic levels of nodes in CMOS digital circuits (i.e. clock and data recovery circuit in WuRx) switch, the electric potential of the silicon substrate changes due to the capacitive coupling between the drains of MOSFETs and the substrate. The potential change propagates to the ana$\log$ frontend circuit through the substrate and acts as "noise" $[8,9]$. The noise fluctuates the threshold voltages of MOSFETs (back-gate effect) in the analog circuits. This is a critical issue for the analog circuits, especially those operating near the threshold voltage to achieve very low 
power consumption. The substrate noise coupling also has an impact on the electric potential in the analog input port. While the signal line in the microstrip structure of the impedance matching circuit is shielded from the silicon substrate by means of the bottom-layer metal, the electric potentials of spiral inductors and metal-insulator-metal (MIM) capacitors may be influenced by the substrate noise through the capacitive coupling [10].

In this paper, the substrate noise coupling between the digital circuit and the input port of the envelop detector in a previously fabricated $2.4-\mathrm{GHz}$ WuRx was investigated experimentally. The propagation path of the substrate noise coupling was clarified by comparing the experimental results with the circuit simulations on the basis of an equivalent circuit model. A modified version of a WuRx to suppress the substrate noise coupling was designed and fabricated. The experimental results for the fabricated WuRx confirmed the effectiveness of the noise coupling suppression recipe.

\section{Evaluation of substrate noise coupling}

Figure 1 illustrates the block diagram of the WuRx used for investigating the substrate coupling noise. The WuRx was fabricated by using $0.18-\mu \mathrm{m}$ mixed signal/RF CMOS process with one poly and six metal layers. It consisted of an impedance matching circuit, an envelope detector, highgain baseband amplifiers, and a clock and data recovery (CDR) circuit. Two spiral inductors and one MIM capacitor were employed in the impedance matching circuit. The envelope detector and baseband amplifiers had the same circuit configuration. The detector operated at around the subthreshold voltage of the MOSFET M1. The detection scheme was based on the nonlinearity of drain current in

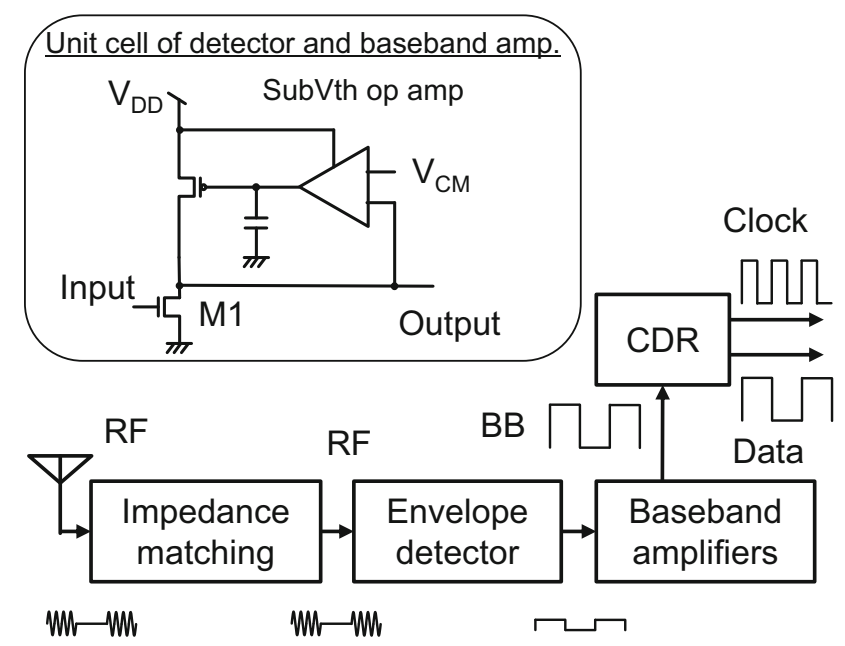

Fig. 1 Block diagram of WuRx the operation bias $[4,5]$. The high-gain baseband amplifiers and detector canceled a DC offset with a subthresholdoperated operational amplifier (subVth op amp) operating as a feedback circuit [11]. The feedback circuit fixed the voltages of the input and output ports of the subVth op amp to common-mode voltage $V_{C M}$ in a low-frequency region to DC. The high-gain baseband amplifiers comprised two parts of the unit cell shown in Fig. 1. The CDR used an injection locked oscillator (ILO) for clock recovery [5]. While the ILO synchronized to the input baseband signal of non-return-to-zero (NRZ) data detected and amplified in the preceding circuits, it was free-running without an input baseband signal. The baseband NRZ data with reduced timing jitter was obtained by latching the baseband signal with a D-type flip/flop and recovered clock.

Figure 2 shows the circuit configuration of the impedance matching circuit, bias circuit, and envelop detector. The input port $I_{D E T}$ was connected to an external current source for simultaneously biasing the gate of MOSFET M1 and the common-mode voltage $V_{C M}$ of the subVth op amp. The voltage fluctuations of the RF input port $\left(R F_{i n}\right)$ and $I_{D E T}$ in the WuRx were measured with a spectrum analyzer when the detector and high-gain amplifiers were turned off and the ILO was free-running. Figure 3 shows the measured spectrum of the detector input port along with that of the free-running clock. A clear coupling of $-73 \mathrm{~dB}$ between the clock output and $I_{D E T}$ was observed, while the voltage fluctuation of $R F_{\text {in }}$ was smaller than the noise floor of the spectrum analyzer. Since the designed value of total gain of the detector and high-gain baseband amplifiers was about $100 \mathrm{~dB}$, a positive feedback loop could be formed. This might result in unwanted oscillation. No correct operation was obtained for the WuRx when the detector and high-gain amplifiers were turned on. To further investigate the coupling between the clock output port and $I_{D E T}$, a square wave with a voltage swing of $1.5 \mathrm{~V}$ and a repetition rate of $377 \mathrm{kHz}$ was applied to the clock output port while the WuRx was turned off. Figure 4 shows the waveforms of $I_{D E T}$ and the applied square wave observed

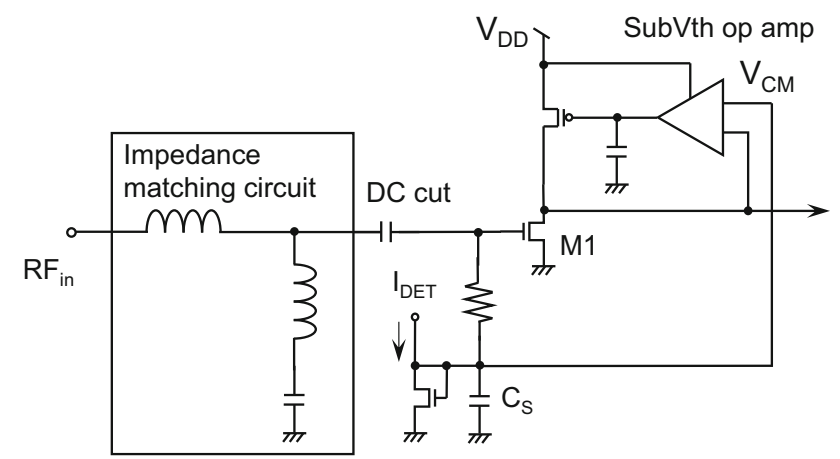

Fig. 2 Circuit configuration of impedance matching circuit, bias circuit, and envelop detector 


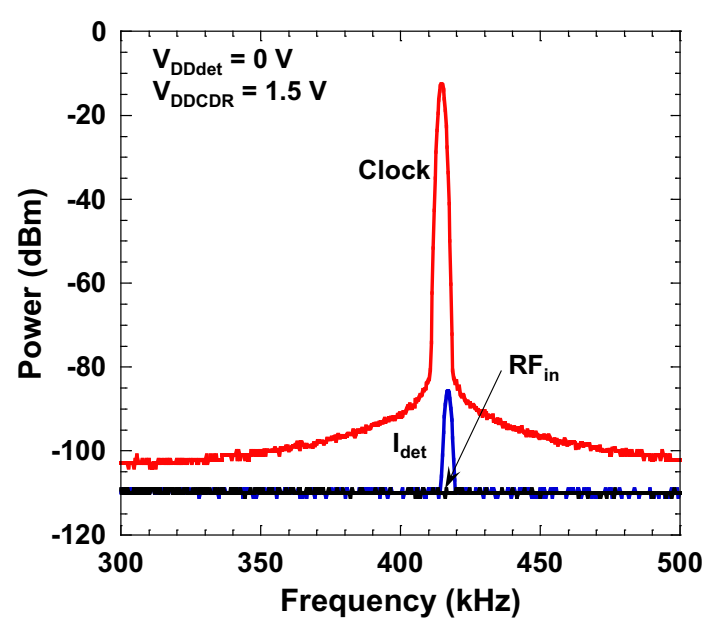

Fig. 3 Measured spectra of detector input port and free-running clock

with an oscilloscope. The waveform of $I_{D E T}$ differentiated from the applied square wave, followed by some ringing wave.

The measured results were compared with circuit simulations to explore the coupling between the clock output port and $I_{D E T}$. Figure 5 shows the schematic circuit model for the simulations. The equivalent circuit models of the spiral inductors and MIM capacitors with parasitic elements were provided by the foundry. The contact resistances of the guard rings and MOSFETs to the substrate were calculated with an elliptic approximation $[12,13]$. Figure 6 shows the simulated waveforms at $I_{D E T}$ and the clock output port, corresponding to those shown in Fig. 4. The circuit simulations revealed that the main coupling path between the clock output port and the input port $I_{D E T}$ was from the MOSFET drains in the clock output buffer to MIM capacitor $C_{S}$ shown in Fig. 5 through the parasitic capacitances and the substrate resistance. Generic logic MOSFETs were used in the clock output buffer and the CDR circuit, and the MIM capacitor $C_{S}$ was placed next to the clock output buffer.

\section{Modified design}

We modified the design of the WuRx on the basis of the findings described in the previous section to suppress the substrate noise coupling. The baseband signal format was changed from NRZ to pulse-width modulation (PWM). In accordance with the signal format, a clock recovery circuit for the PWM signal [4] was used. Since the repetition frequency of a ring oscillator in the clock recovery circuit was one-order higher than that of baseband signal frequency [4], the gain of the baseband amplifier was decreased at the repetition frequency of the ring oscillator. Generic logic MOSFETs in the data and clock buffers were replaced by deep N-well (DNW) RF MOSFETs, and microstrip lines were used between the CDR circuit and output buffers to reduce the noise current injection to the substrate. The capacitance value of $C S$ was decreased from 10 to $1 \mathrm{pF}$ to reduce the parasitic capacitance between the MIM capacitor and the substrate. The CDR circuit was surrounded by a grounded DNW guard ring to reduce the
Fig. 4 Waveforms of input port IDET and applied square wave

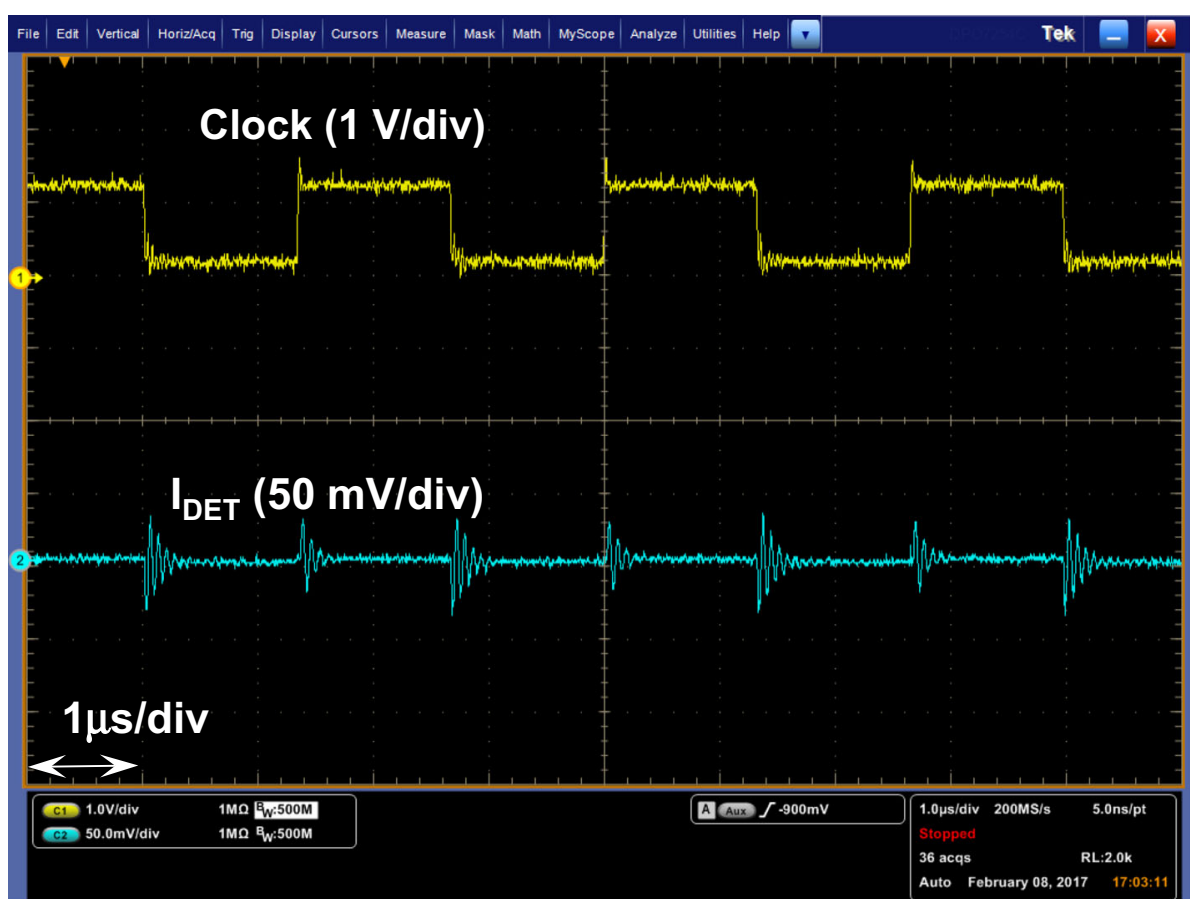


Fig. 5 Schematic circuit model for simulating substrate noise coupling

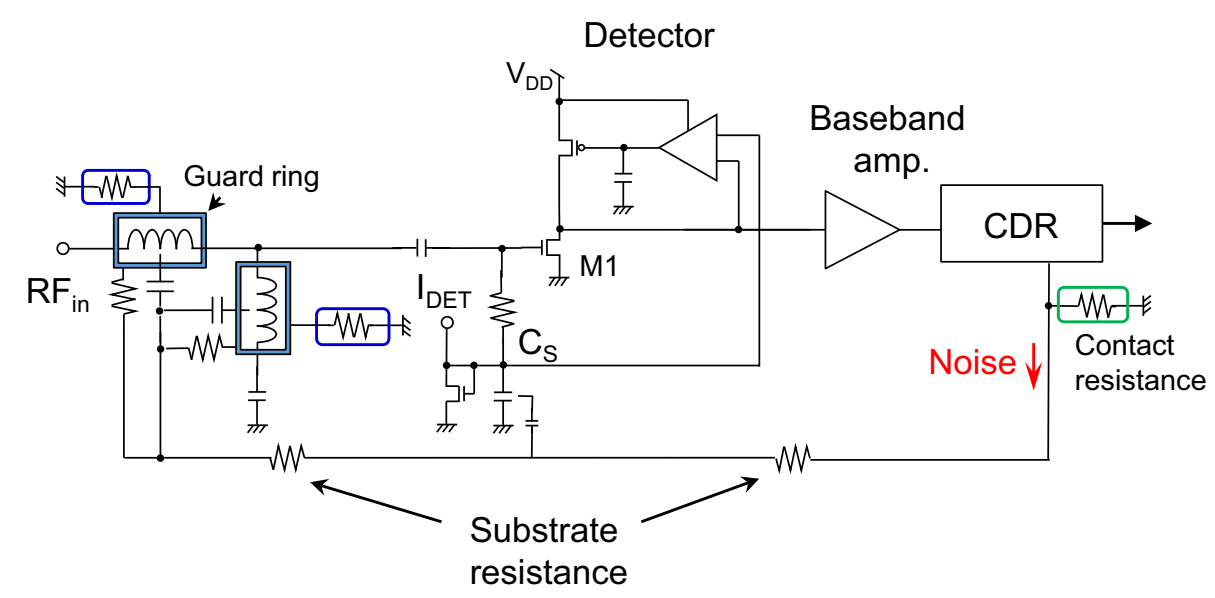

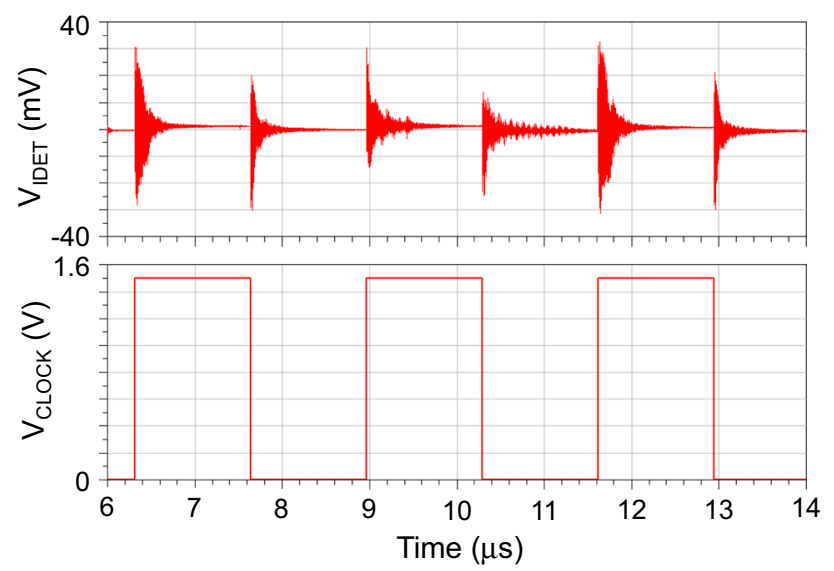

Fig. 6 Simulated waveforms of input port IDET and clock output port

noise current injection [14], and only one spiral inductor was used in the impedance matching circuit.

\section{Experimental results}

A modified version of the WuRx was fabricated with the same CMOS process as the previous version. Figure 7 shows a microphotograph of the fabricated WuRx. The fabricated WuRx was measured with RF and power-supply probes on a chip. The logic " 1 " and " 0 " in the PWM format were defined as pulses with duty ratios of 75 and $25 \%$, respectively. A NRZ-to-PWM converter was fabricated with commercially available logic ICs based on the definition. A 2.4-GHz continuous wave $(\mathrm{CW})$ was modulated with the PWM baseband signal converted from a NRZ pseudo-random bit stream, and input to the WuRx. Figure 8 shows the measured waveforms. The uppermost wave was a 100-kbps PWM baseband signal used to modulate 2.4-GHz CW. Successful recovery of NRZ data and clock from a PWM-modulated signal with power of

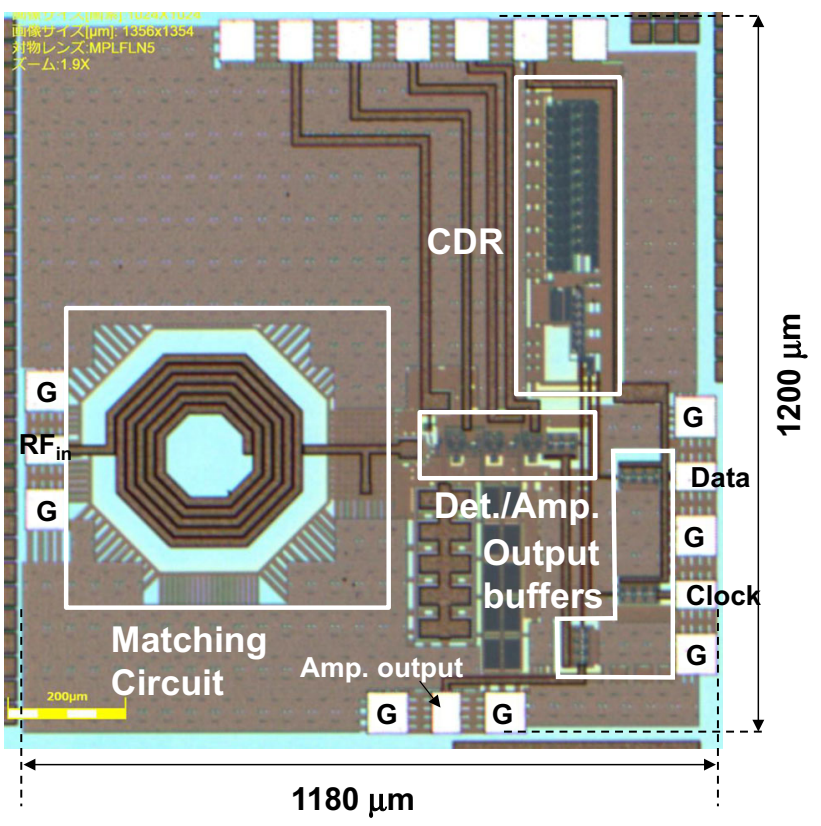

Fig. 7 Microphotograph of fabricated WuRx

$-30 \mathrm{dBm}$ was achieved. Sensitivity of our circuit at a bit error rate (BER) of $10^{-3}$ was $-32 \mathrm{dBm}$. Also, the sensitivity estimated from noise and conversion efficiency [1] is given to

Sensitivity $=\frac{3.5 \sqrt{\int N(f) d f}}{\gamma}$,

where $N(f)$ is noise power density, and $\gamma$ is conversion efficiency [output voltage/input power (V/W)]. The calculated sensitivity using Eq. (1) was $-46 \mathrm{dBm}$. We consider that the reason why the BER degraded is due to noise of the measurement setup. The power consumption of the detector and baseband amplifiers was $11.3 \mu \mathrm{W}$, while that of the CDR circuit including data and clock output buffers was $83.7 \mu \mathrm{W}$. 
Fig. 8 Measured waveforms of 100-kbps PWM baseband signal used to modulate $2.4-\mathrm{GHz} \mathrm{CW}$, recovered NRZ data, and clock

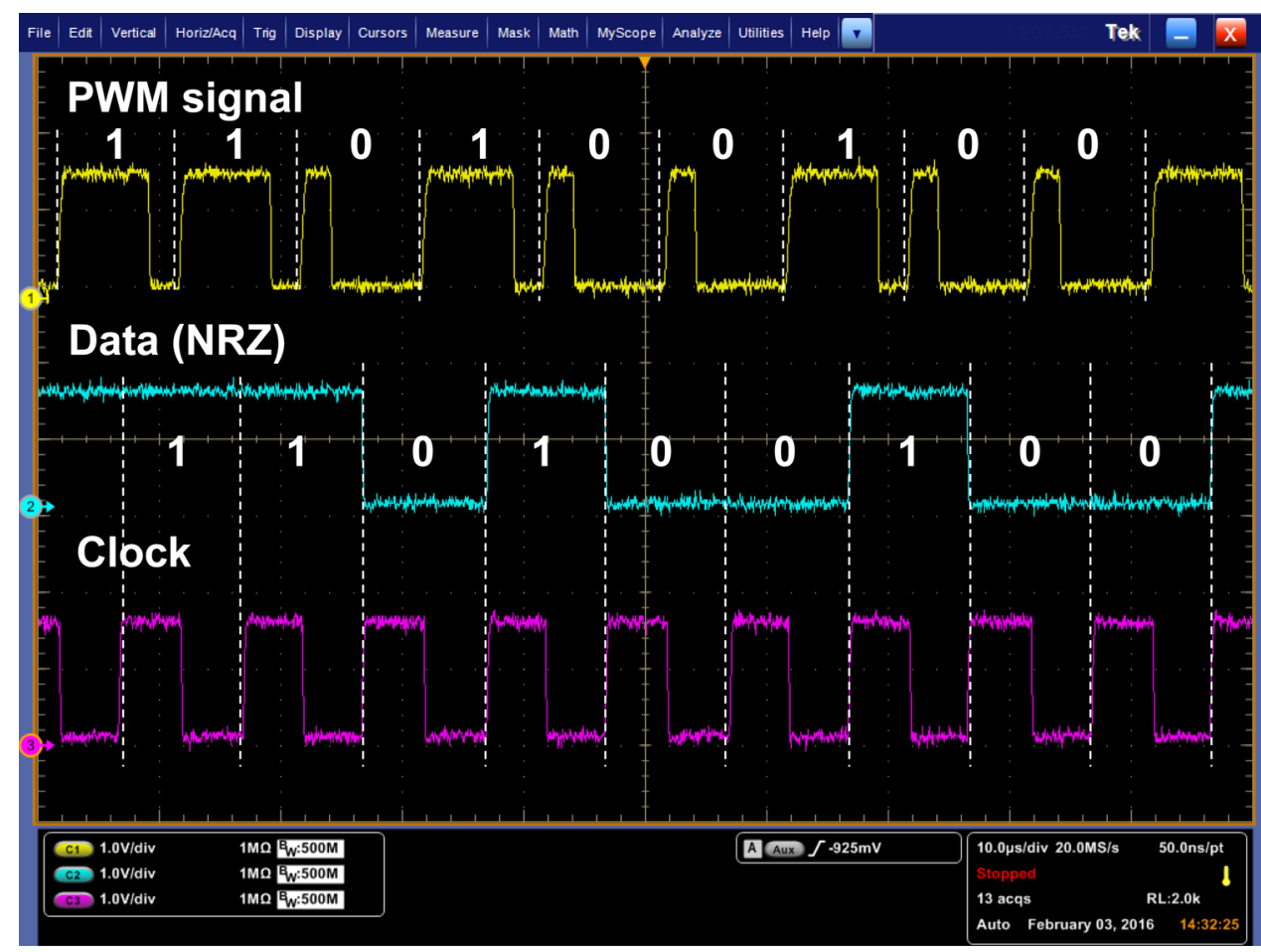

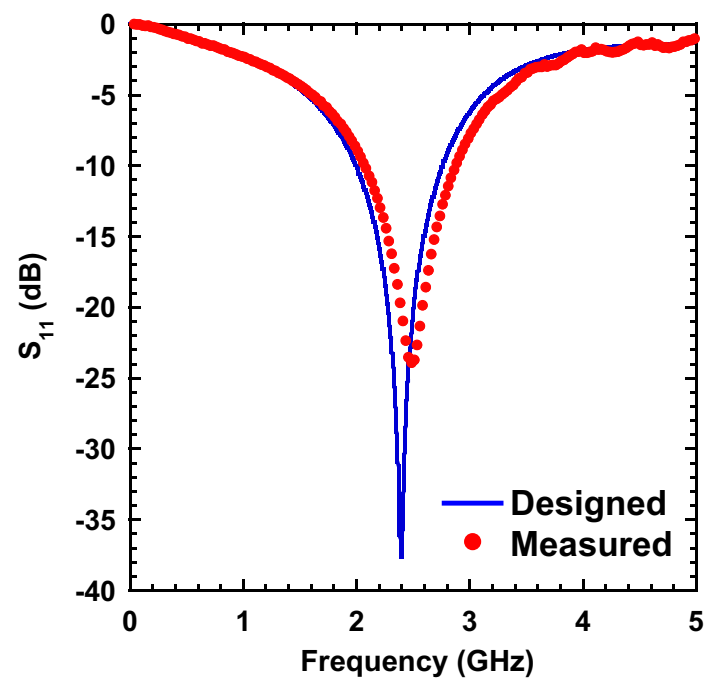

Fig. 9 Return loss S11 of WuRx

Figure 9 shows the return loss S11 of the WuRx measured with a vector network analyzer. The measured S11 matched the designed S11, and good impedance matching of $-20 \mathrm{~dB}$ was achieved at $2.4 \mathrm{GHz}$. Figure 10 shows the waveforms of $I_{D E T}$ and the square wave applied to the clock output port, where the experimental conditions were the same as those described in Sect. 2. The substrate noise coupling in the waveform was successfully suppressed when compared with that in the waveform shown in Fig. 4. Table 1 shows the performance comparison among our prototype circuit of this research and related works. In this research, we achieved to integrate and run the WuRx with a CDR, which is a logic circuit, without mixer, LNA and other extra circuits.

\section{Conclusion}

The substrate noise coupling between the clock recovery circuit and the input port of the envelop detector in a previously fabricated WuRx was investigated experimentally. The propagation path of the substrate noise coupling was clarified by comparing the experimental results with the circuit simulations on the basis of an equivalent circuit model. The design of the WuRx was modified on the basis of the findings to suppress the substrate noise coupling. The fabricated WuRx successfully operated a 100-kbps PWM signal with a carrier frequency of $2.4 \mathrm{GHz}$, and the effectiveness of the noise coupling suppression recipe was confirmed. 
Fig. 10 Waveforms of input port IDET and applied square wave for modified WuRx

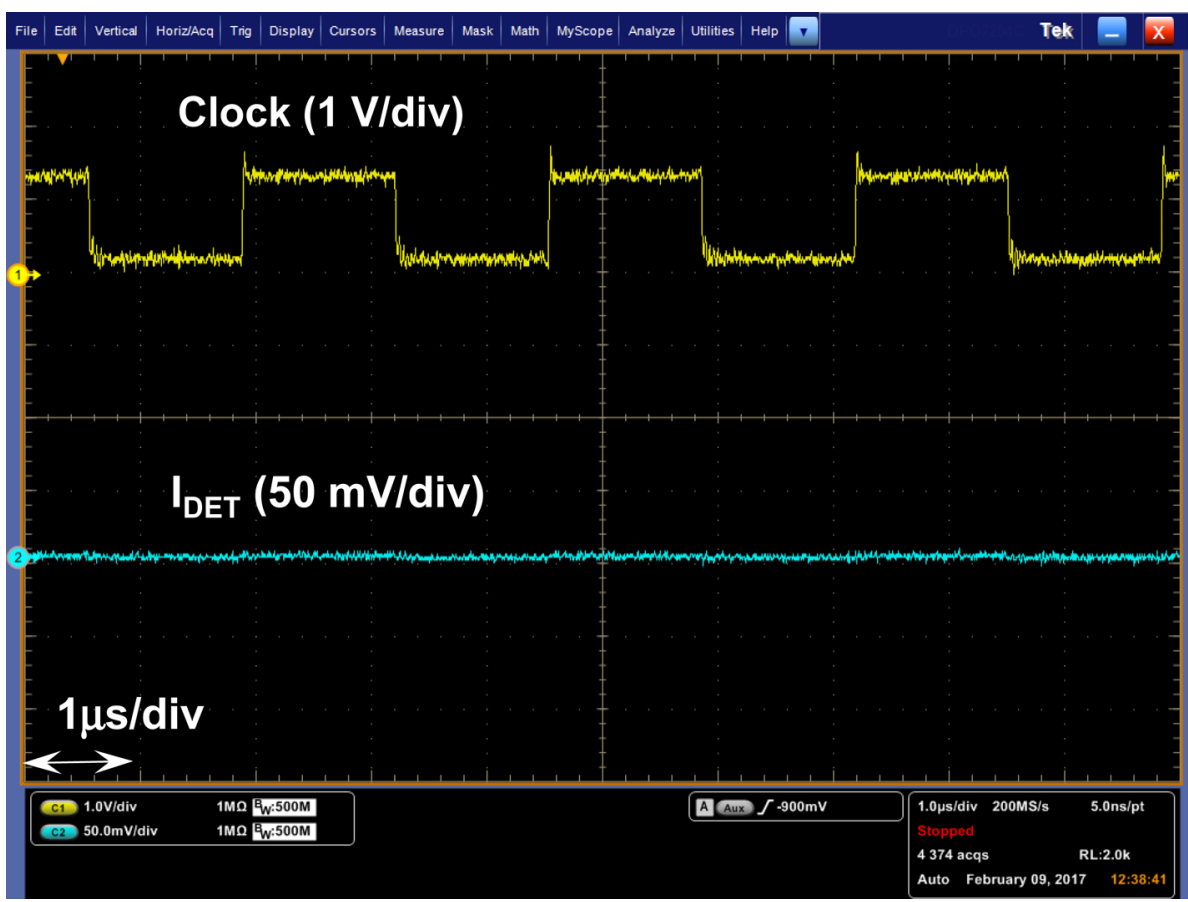

Table 1 Performance comparison of wake-up receivers

\begin{tabular}{|c|c|c|c|c|c|c|c|c|c|}
\hline \multirow[t]{2}{*}{ References } & \multirow[t]{2}{*}{ Power $(\mu \mathrm{W})$} & \multirow[t]{2}{*}{ Carrier (GHz) } & \multirow[t]{2}{*}{ Sensitivity (dBm) } & \multirow[t]{2}{*}{ Bit rate $(\mathrm{kbit} / \mathrm{s})$} & \multirow[t]{2}{*}{ Process (nm) } & \multicolumn{4}{|c|}{ Configuration } \\
\hline & & & & & & Mixer & LNA & CDR & External component \\
\hline [2] & 52 & 2 & $-72^{\mathrm{a}}$ & 100 & 90 & Yes & No & No & Yes \\
\hline$[3]$ & 51 & $0.915 / 2.4$ & $-75 /-64^{b}$ & 100 & 90 & No & Yes & No & No \\
\hline$[15]$ & 415 & 2.4 & $-82^{\mathrm{a}}$ & 500 & 65 & Yes & No & No & Yes \\
\hline [16] & 2.4 & 0.868 & $-71^{\mathrm{a}}$ & 100 & 130 & No & Yes & No & Yes \\
\hline [4] & 6.8 & 1.75 & $-47.2^{\mathrm{b}}$ & 1 & 180 & No & No & No & No \\
\hline$[5]$ & 9 & 60 & $-68^{b}$ & 350 & 180 & No & No & Yes & No \\
\hline$[6]$ & 4.5 & 2.4 & $-50^{\mathrm{a}}$ & 100 & 180 & No & No & No & Yes \\
\hline This work & 11.3 & 2.4 & $-32^{\mathrm{a}} /-46^{\mathrm{b}}$ & 100 & 180 & No & No & Yes & No \\
\hline
\end{tabular}

${ }^{\mathrm{a}}$ Estimated at a bit error rate (BER) of $10^{-3}$

${ }^{\mathrm{b}}$ Estimated from noise and conversion efficiency

Acknowledgements This work was partially supported by the Ministry of Internal Affairs and Communications of Japan/Strategic Information and Communications R\&D Promotion Programme (MIC/ SCOPE) \#151301001 and the VLSI Design and Education Center (VDEC), University of Tokyo in collaboration with Cadence Design System, Inc. and Keysight Technologies Japan, Ltd.

Open Access This article is distributed under the terms of the Creative Commons Attribution 4.0 International License (http://creative commons.org/licenses/by/4.0/), which permits unrestricted use, distribution, and reproduction in any medium, provided you give appropriate credit to the original author(s) and the source, provide a link to the Creative Commons license, and indicate if changes were made.

\section{References}

1. Kolinko, P., \& Larson, L. E. (2007). Passive RF receiver design for wireless sensor networks. In IEEE MTT-S international microwave symposium, Honolulu (pp. 567-570).

2. Pletcher, N. M., Gambini, S., \& Rabaey, J. (2009). A $52 \mu \mathrm{w}$ wake-up receiver with $-72 \mathrm{dBm}$ sensitivity using an uncertainIF architecture. IEEE Journal of Solid-State Circuits, 44(1), 269-280.

3. Drago, S., Leenaerts, D. M. W., Sebastiano, F., Breems, L. J., Makinwa, K. A. A., \& Nauta, B. (2010). A 2.4 GHz 830 pJ/bit duty-cycled wake-up receiver with $-82 \mathrm{dBm}$ sensitivity for crystal-less wireless sensor nodes. In International solid-state circuits conference, San Francisco (pp. 224-225).

4. Takahagi, K., Matsushita, H., Iida, T., Ikebe, M., Amemiya, Y., \& Sano, E. (2013). Low-power wake-up receiver with 
subthreshold CMOS circuits for wireless sensor networks. Analog Integrated Circuits and Signal Processing, 75, 199-205.

5. Wada, T., Ikebe, M., \& Sano, E. (2013). 60-GHz, 9- $\mu$ W wake-up receiver for short-range wireless communications. In European solid-state circuits conference, Bucharest (pp. 383-386).

6. Chen, S. E., Yang, C. L., \& Cheng, K. W. (2015). A $4.5 \mu$ w 2.4 $\mathrm{GHz}$ wake-up receiver based on complementary current-reuse RF detector. In IEEE international symposium on circuits and systems, Lisbon (pp. 1214-1217).

7. Magno, M., Jelicic, V., Srbinovski, B., Bilas, V., Popovici, E., \& Benini, L. (2016). Design, implementation, and performance evaluation of a flexible low-latency nanowatt wake-up radio receiver. IEEE Transactions on Industrial Informatics, 12(2), 633-644.

8. Su, D. K., Loinaz, M. J., Masui, S., \& Wooley, B. A. (1993). Experimental results and modeling techniques for substrate noise in mixed-signal integrated circuits. IEEE Journal of Solid-State Circuits, 28(4), 420-430.

9. Verghese, N. K., \& Allstot, D. J. (1998). Computer-aided design considerations for mixed-signal coupling in RF integrated circuits. IEEE Journal of Solid-State Circuits, 33(3), 314-323.

10. Pun, A. L. L., Yeung, T., Lau, J., Clement, F. J. R., \& Su, D. K. (1998). Substrate noise coupling through planar spiral inductor. IEEE Journal of Solid-State Circuits, 33(6), 877-884.

11. Iida, T., Asai, T., Sano, E., \& Amemiya, Y. (2009). Offset cancellation with subthreshold-operated feedback circuit for fully differential amplifiers. In Proceedings of the 16th IEEE international conference on electronics, circuits, and systems, Tunisia (pp. 140-143).

12. Kristiansson, S., Ingvarson, F., \& Jeppson, K. O. (2005). Modeling of rectangular contacts for noise coupling analysis in homogeneous substrates. In Norchip conference, Oulu (pp. 24-27).

13. Kristiansson, S., Ingvarson, F., \& Jeppson, K. O. (2007). Compact spreading resistance model for rectangular contacts on uniform and epitaxial substrates. IEEE Transactions on Electron Devices, 54(9), 2531-2536.

14. Kosaka, D., Nagata, M., Hiraoka, Y., Imanishi, I., Maeda, M., Murasaka, Y., et al. (2005). Isolation strategy against substrate coupling in CMOS mixed-signal/RF circuits. In Symposium VLSI circuits digest technical papers, Kyoto (pp. 276-279).

15. Kolinko, P., \& Larson, L. E. (2007). Passive RF receiver design for wireless sensor networks. In IEEE MTT-S international microwave symposium, Honolulu (pp. 567-570).

16. Huang, X., Rampu, S., Wang, X., Dolmans, G., \& Groot, H. (2010). A $2.4 \mathrm{GHz} / 915 \mathrm{MHz} 51 \mu \mathrm{W}$ wake-up receiver with offset and noise suppression. In International solid-state circuits conference, San Francisco (pp. 222-223).

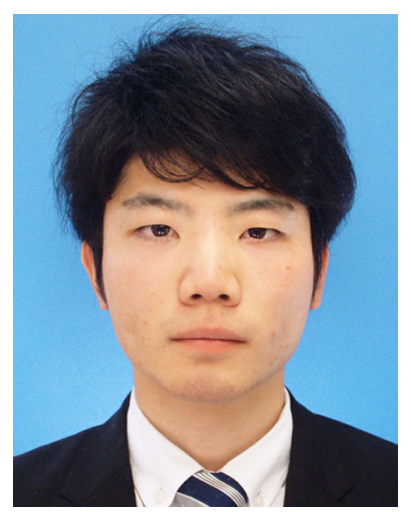

Shota Hiramatsu received B.S. degrees in electrical engineering from Hokkaido University, Sapporo, Japan, in 2016. He is currently pursuing the M.S. degree in electrical engineering at Hokkaido University. His current research interests is design of CMOS RF/millimeterwave circuits and ultra-low power circuits for wireless sensor networks.

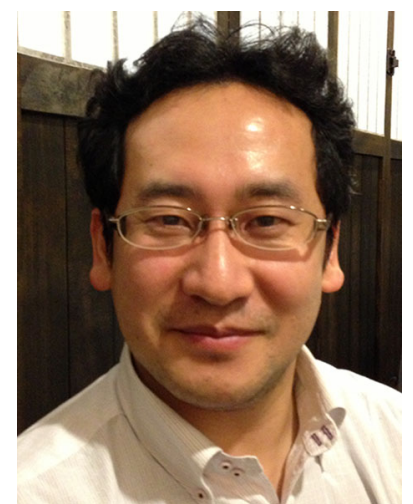

Masayuki Ikebe received B.S., M.S. and Ph.D. degrees in electrical engineering from Hokkaido University, Hokkaido, Japan, in 1995, 1997 and 2000, respectively. During 2000-2004, he worked for the Electronic Device Laboratory, Dai Nippon Printing Corporation, Akabane, Japan, where he was engaged in the research and development of wireless communication system and image processing system. Presently, he is a Professor of the Research Center for Integrated Quantum Electronics, Hokkaido University. His current research includes CMOS RF circuits and analog circuits.

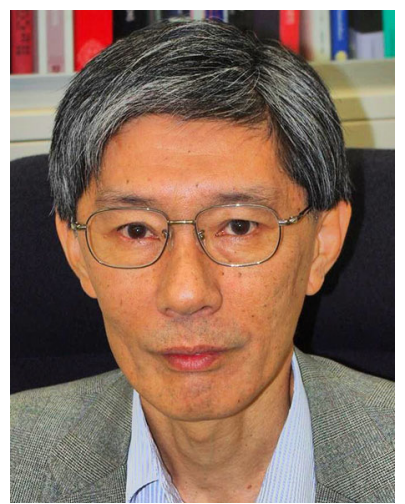

Eiichi Sano was born in Shizuoka, Japan, in 1952. He received B.S., M.S., and Ph.D. degrees from the University of Tokyo, Japan, in 1975, 1977, and 1998, respectively. From 1977 to 2001, he was with NTT laboratories, where he worked on MOS device physics, mixed analog/digital MOS ULSIs, ultrafast MSM photodetectors, electrooptic sampling, highspeed electronic and optoelectronic ICs. In 2001, he joined the Research Center for Integrated Quantum Electronics, Hokkaido University, as a professor. His current research interests include nanocarbon materials, terahertz devices, and CMOS integrated circuits. He has published over 300 papers in major journals and conference proceedings related to these research areas. Dr. Sano is a fellow of the Institute of Electronics, Information and Communication Engineers of Japan and a member of the Japan Society of Applied Physics.

Publisher's Note Springer Nature remains neutral with regard to jurisdictional claims in published maps and institutional affiliations. 